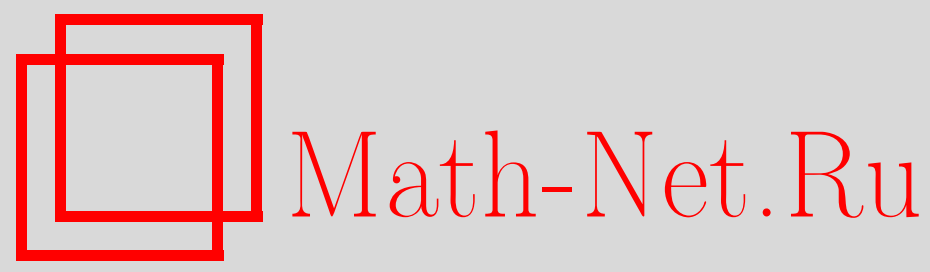

А. И. Павлов, Аналитическая непродолжаемость степенных рядов некоторых классов, Матем. заметки, 1998, том 64, выпуск 3, 474-477

DOI: https://doi.org/10.4213/mzm1421

Использование Общероссийского математического портала Math-Net.Ru подразумевает, что вы прочитали и согласны с пользовательским соглашением http://www . mathnet.ru/rus/agreement

Параметры загрузки:

IP : 18.209 .158 .208

26 апреля 2023 г., $14: 22: 43$

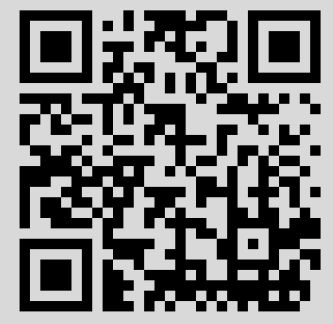




\section{АНАЛИТИЧЕСКАЯ НЕПРОДОЛЖАЕМОСТЬ СТЕПЕННЫХ РЯДОВ НЕКОТОРЫХ КЛАССОВ}

\section{А. И. Павлов}

Целью настоящей публикации является доказательство следующих утверждений.

Теорема 1. Пусть действительное иррациональное число $\alpha>0$ и последовательность натуральных чисел $M$ удовлетворяет условиям:

1) плотность последовательности $M$ полохительна, т.е.

$$
\lim _{N \rightarrow \infty} \frac{1}{N} \sum_{\substack{m \leqslant N \\ m \in M}} 1=\gamma>0 ;
$$

2) последовательность $a_{\nu}=\left\{\alpha m_{\nu}\right\}, \nu=1,2, \ldots, m_{\nu} \in M$, равномерно распределена

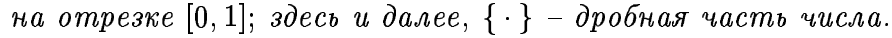

Тогда если $f(x)$ - интегрируемая по Риману на отрезке [0,1] функиия, коэффициенты Фурье с достаточно большими (по модулю) номерами которой не равны 0 , то степенной ряд

$$
F(z)=\sum_{m \in M} f(\{\alpha m\}) z^{m}
$$

сходящийся в круге $|z|<1$, не может быть аналитически продолжен в область $|z|>1$ через любую дугу на окружности $|z|=1$.

ТеОРема 2. Пусть число $\alpha$ и последовательность $М$ удовлетворяют условиям теоремы 1,

$$
P(x, y)=\sum_{\nu=0}^{k} x^{\nu} p_{\nu}(y),
$$

где челое $k \geqslant 1, p_{\nu}(y)$ - многочлен с комплексными коэффичиентами, $\nu=0,1, \ldots, k, u$ $p_{k}(y) \not \equiv C$, где $C$ - постоянная. Тогда степенной ряд

$$
F(z)=\sum_{m \in M} P(m,\{\alpha m\}) z^{m}
$$

сходящийся в круге $|z|<1$, не может бъть аналитически продолжен в область $|z|>1$ через любую дугу на окружности $|z|=1$.

ЗАмЕчаниЕ 1 . В случае, когда последовательность $M$ совпадает с множеством всех натуральных чисел, обе теоремы были доказаны Морделлом в работах [1] и [2].

ЗАмечАние 2. В качестве $M$ можно рассматривать, например, следующие множества натуральных чисел:

1) арифметическая прогрессия $m_{\nu}=a \nu+b, \nu=1,2 \ldots$, где целые $a$ и $b$ такие, что $a \geqslant 1$ и $b \geqslant 0$

2 ) числа без $k$-х степеней, $k \geqslant 2$; напомним, что натуральное число $m$ называется числом $6 е з$ $k$ - $x$ cтепеней, если для любого простого $p$ выполнено условие $p^{k} \nmid m$;

3 ) обобщение чисел без $k$-х степеней (см. [3]).

Работа выполнена при финансовой поддержке Российского фонда фундаментальных исследований, грант № 97-01-00721. 
ЗАмечаниЕ 3 . Если $\gamma=0$ и множество $M$ бесконечно, то теоремы 1 и 2 следуют из теоремы Фабри [4].

Доказательство теоремы 1 опирается на критерий $\Gamma$. Вейля [5]: последовательность $a_{n}, n=1$, $2, \ldots$, действительных чисел равномерно распределена на отрезке $[0,1]$ тогда и только тог да, когда для любой интегрируемой по Риману на отрезке $[0,1]$ функции $\varphi(x)$ выполняется равенство

$$
\lim _{N \rightarrow \infty} \frac{1}{N} \sum_{n=1}^{N} \varphi\left(a_{n}\right)=\int_{0}^{1} \varphi(x) d x
$$

ДОКАЗАТЕЛЬСТВО ТЕОРЕМЫ 1 . Пусть целое $h \neq 0$ и $0<r<1$. Тогда

$$
\begin{aligned}
F\left(r e^{2 \pi i \alpha h}\right) & =(1-r)\left(\sum_{m=0}^{\infty} r^{m}\right)\left(\sum_{m \in M} f(\{\alpha m\}) e^{2 \pi i \alpha h m} r^{m}\right) \\
& =(1-r) \sum_{N=1}^{\infty} r^{N} \sum_{\substack{m \leqslant N \\
m \in M}} f(\{\alpha m\}) e^{2 \pi i \alpha h m} .
\end{aligned}
$$

Согласно критерию Г. Вейля и условию теоремы 1

$$
\lim _{N \rightarrow \infty} \frac{1}{N} \sum_{\substack{m \leqslant N \\ m \in M}} f(\{\alpha m\}) e^{2 \pi i \alpha h m}=\gamma \widehat{f}(h)
$$

где $\widehat{f}(h)-$ коэффициент Фурье с номером $h$ функции $f(x)$ на отрезке [0,1]. Следовательно, при $0<r<1$ и $r \rightarrow 1$

$$
F\left(r e^{2 \pi i \alpha h}\right)=(1-r) \gamma f(h) \sum_{N=1}^{\infty} N r^{N}(1+o(1))=\frac{\gamma \widehat{f}(h)}{1-r}+o\left(\frac{1}{1-r}\right)
$$

Согласно условию теоремы 1 существует $h_{0}>0$ такое, что при $|h|>h_{0}$ выполняется неравенство $f(h) \neq 0$. Поэтому при $|h|>h_{0}$ и $r<1$ получим

$$
\lim _{r \rightarrow 1}\left|F\left(r e^{2 \pi i \alpha h}\right)\right|=\infty
$$

Значит, точка $z_{h}=e^{2 \pi i \alpha h}$ является особой точкой для функции $F(z)$. Так как множество точек $z_{h}$, когда $h$ пробегает множество всех целых чисел, всюду плотно на окружности $|z|=1$, теорема 1 доказана.

Для доказательства теоремы 2 требуется утверждение, которое, возможно, известно.

ЛЕмма. Если для некоторого многочлена $Q(x)$ степени $k \geqslant 1$ найдутся челые попарно различные числа $h_{1}, h_{2}, \ldots, h_{k}$ такие, что

$$
\int_{0}^{1} Q(x) e^{2 \pi i x h_{\nu}} d x=0, \quad h_{\nu} \neq 0, \quad \nu=1,2, \ldots, k
$$

то выполняется тохсество $Q(x) \equiv Q(0)$. 
ДоКАЗАТЕЛЬСТвО. Пусть

$$
Q(x)=\sum_{m=0}^{k} Q_{m} x^{m},
$$

где $Q_{0}, Q_{1}, \ldots, Q_{k}$ - комплексные числа. Тогда, пользуясь равенством $(h \neq 0$ - целое $)$

$$
\int_{0}^{1} Q(x) e^{2 \pi i x h} d x=\sum_{m=0}^{k}(-1)^{m} \frac{Q^{(m)}(1)-Q^{(m)}(0)}{(2 \pi i h)^{m+1}}
$$

где $Q^{(m)}(x)$ - производная порядка $m$ многочлена $Q(x)$, и равенствами $(2)$, получим однородную систему линейных уравнений относительно разностей $Q^{(m)}(1)-Q^{(m)}(0), m=1,2, \ldots, k$,

$$
\sum_{m=0}^{k}(-1)^{m} \frac{Q^{(m)}(1)-Q^{(m)}(0)}{\left(2 \pi i h_{\nu}\right)^{m+1}}=0, \quad \nu=1,2, \ldots, k .
$$

Если $\Delta$ - определитель этой системы, то $\Delta=\left(h_{1} \cdots h_{k}\right)^{-1} W\left(h_{1}^{-1}, \ldots, h_{k}^{-1}\right)$, где $W\left(h_{1}, \ldots, h_{k}\right)-$ определитель Вандермонда, построенный на системе чисел $h_{1}, \ldots, h_{k}$. Согласно условию леммы получим $W\left(h_{1}, \ldots, h_{k}\right) \neq 0$. Значит, система (3) имеет только тривиальное решение:

$$
Q^{(m)}(1)-Q^{(m)}(0)=0, \quad m=1,2, \ldots, k .
$$

Так как $Q^{(m)}(0)=m ! Q_{m}$ и

$$
Q_{m}^{(1)}=\sum_{\nu=m}^{k} \nu(\nu-1) \cdots(\nu-m+1) Q_{\nu}, \quad m=0,1, \ldots, k,
$$

получим систему уравнений относительно чисел $Q_{1}, \ldots, Q_{k}$

$$
\sum_{\nu=m}^{k} \nu(\nu-1) \cdots(\nu-m+1) Q_{\nu}=m ! Q_{m}, \quad m=0,1, \ldots, k
$$

Из уравнения с номером $m=k-1$ получим $Q_{k}=0$. Подставляя $Q_{k}=0$ в уравнение (4) с номером $m=k-2$, получим $Q_{k-1}=0$. Рассуждая аналогично, получим $Q_{k-2}=Q_{k-3}=\cdots=Q_{1}=0$.

ДоКАЗАТЕЛЬСТвО ТЕОРЕМЫ 2 . Пусть целое $h \neq 0$ и $0<r<1$. Тогда при $r \rightarrow 1$

$$
F\left(r e^{2 \pi i \alpha h}\right)=\sum_{m \in M} P(m,\{\alpha m\}) e^{2 \pi i \alpha h m} r^{m}=\sum_{m \in M} m^{k} p_{k}(\{\alpha m\}) e^{2 \pi i \alpha h m} r^{m}+O\left((1-r)^{-k}\right) .
$$

Далее,

$$
\sum_{m \in M} m^{k} p_{k}(\{\alpha m\}) e^{2 \pi i \alpha h m} r^{m}=(1-r) \sum_{N=1}^{\infty} r^{N} \sum_{\substack{m \leqslant N \\ m \in M}} m^{k} p_{k}(\{\alpha m\}) e^{2 \pi i \alpha h m}
$$

Если

$$
S_{\alpha, h}(N)=\sum_{\substack{m \leqslant N \\ m \in M}} p_{k}(\{\alpha m\}) e^{2 \pi i \alpha h m}, \quad N \geqslant 1
$$


топри фиксированном $h \neq 0$ и $N \rightarrow \infty$ согласно критерию Вейля получим асимптотику $S_{\alpha, h}(N) \sim$ $\gamma \widehat{p}_{k}(h) N$, где $\gamma$-плотность последовательности $M$. Значит, при $N \rightarrow \infty$ и $S_{\alpha, h}(0)=0$ получим

$$
\begin{aligned}
\sum_{\substack{m \leqslant N \\
m \in M}} m^{k} p_{k}(\{\alpha m\}) e^{2 \pi i \alpha h m} & =\sum_{m=1}^{N} m^{k}\left(S_{\alpha, h}(m)-S_{\alpha, h}(m-1)\right) \\
& =\sum_{m=1}^{N-1}\left(m^{k}-(m+1)^{k}\right) S_{\alpha, h}(m)+N^{k} S_{\alpha, h}(N) \\
& =N^{k} S_{\alpha, h}(N)-k \sum_{m=1}^{N} m^{k-1} S_{\alpha, h}(m)+O\left(N^{k}\right) \\
& =\gamma \widehat{p}_{k}(h) N^{k+1}-\gamma \widehat{p}_{k}(h) k \sum_{m=1}^{N} m^{k}+o\left(N^{k+1}\right) \\
& =\frac{\gamma \widehat{p}_{k}(h)}{k+1} N^{k+1}+o\left(N^{k+1}\right) .
\end{aligned}
$$

Следовательно, из (5) и (6) при $r \rightarrow 1$ получим

$$
F\left(r e^{2 \pi i \alpha h}\right)=\frac{\gamma \widehat{p}_{k}(h)}{k+1}(1-r)^{-(k+1)}+o\left((1-r)^{-(k+1)}\right) .
$$

Так как $p_{k}(y) \not \equiv p_{k}(0)$, согласно лемме существует $h_{0}>0$ такое, что $\widehat{p}_{k}(h) \neq 0$ при $|h|>h_{0}$. Следовательно, при таких $h$ из (7) следует равенство (1).

Значит, точка $z_{h}=e^{2 \pi i \alpha h}$ для функции $F(z)$ является особой. Так как множество точек $z_{h}$ при $|h|>h_{0}$ всюду плотно на окружности $|z|=1$, теорема 2 доказана.

\section{СПИСОК ЦИТИРОВАННОЙ ЛИТЕРАТУРЫ}

1. Mordell L. J. // Proc. Amer. Math. Soc. 1961. V. 12. P. 522-526. 2. Mordell L. J. // Notices Amer. Math. Soc. 1964. V. 11. Р. 312. 3. Павлов А. И. // Матем. заметки. 1994. Т. 55. № 2. С. 102-108. 4. Бибербах Л. Аналитическое продолжение. М.: Наука, 1967. 5. Кейперс Л., Нидеррейтер Г. Равномерное распределение последовательностей. М.: Наука, 1985. 